\title{
SAND-- $98-8500$ \\ An Atomistic Study of the Effects of Stress and Hydrogen on a Dislocation Lock in Nickel
}

R. G. Hoagland, School of Mechanical and Materials Engineering, Washington State University, Pullman, WA 99164-2920

M. I. Baskes, Materials Reliability Department, Sandia National Laboratories, Livermore, CA 94551-0969

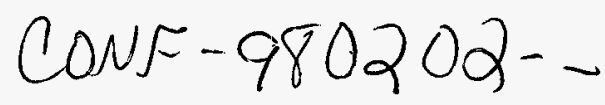

\section{Introduction}

Even though austenitic alloys are commonly used in a hydrogen environment, hydrogeninduced fracture of these alloys has been reported (1-3). Most recently it has been shown that the failure of these alloys in hydrogen is initiated by void formation at slip band intersections (4). It is the object of this work to investigate the atomistic mechanisms that occur at these slip band intersections in the presence of hydrogen. Specifically it has been suggested that dislocation-dislocation interactions may play a large role in the initiation of voids or cracks (5). Hirth (6) has summarized the various forms of dislocation interactions, traditionally called Lomer-Cottrell Locks (LCLs), that can occur. Baskes et al. (7) have investigated the effects of stress on a LCL using an Embedded Atom Method (EAM) model for nickel developed previously by Angelo et al. $(8,9)$. The EAM is a well-established semi-empirical method of atomistic calculation that has been successfully used for over a decade to calculate the energetics and structure of defects in transition metals (10-12). The work by Angelo et al. (8) established that the trapping of hydrogen to single dislocations had a maximum energy of $\sim 0.1 \mathrm{eV}$ while the trapping to a LCL was significantly greater, $\sim 0.33 \mathrm{eV}$, thus we expect that a LCL could be important in explaining the fracture behavior of a fcc material in a hydrogen environment.

Baskes et al. (7) found that under uniaxial stress a LCL in the absence of hydrogen underwent a number of transitions, but it did not dissociate or form a crack nucleus. In this work we extend the previous work to include the effects of hydrogen. Specifically we will simulate the experiments of Moody et al. (4) for the case of room temperature exposure of Inconel to 190 atm of hydrogen.

\section{Computational Procedure}

As mentioned in the introduction, the energetics arise from EAM potentials $(8,9)$. These potentials describe the properties of $\mathrm{Ni}$ and the $\mathrm{H} / \mathrm{Ni}$ interactions quite well. A schematic of the geometry, which is identical to that used in Baskes et al. (7), is shown in Figure 1 which depicts a cylindrical region periodic ( $5 \AA$ period) along the cylinder axis. The initial atom positions are generated using anisotropic elasticity theory for a single $a_{0}[100]$ ( $a_{0}$ is the lattice constant) dislocation at the center of the cylinder with its dislocation line parallel to the axis of the cylinder. The Burgers' vector of this dislocation is the net Burgers' vector of a LCL-3 (6) (see Figure 2). Atoms far from the dislocation core are held fixed at these positions, while atoms in the central region are allowed to relax to their minimum energy positions. Uniaxial stress is applied by incremental $(0.2 \%$ per step) application of a homogeneous strain in the $\mathrm{x}$ ([100]) direction as in Baskes et al. (7).

In order to simulate the external hydrogen environment the following procedure is used as in Baskes et al. (13):

1) The equilibrium lattice constant is obtained through Monte Carlo (MC) calculations with dynamic periodic boundaries of a bulk nickel lattice at $295 \mathrm{~K}$ at zero pressure.

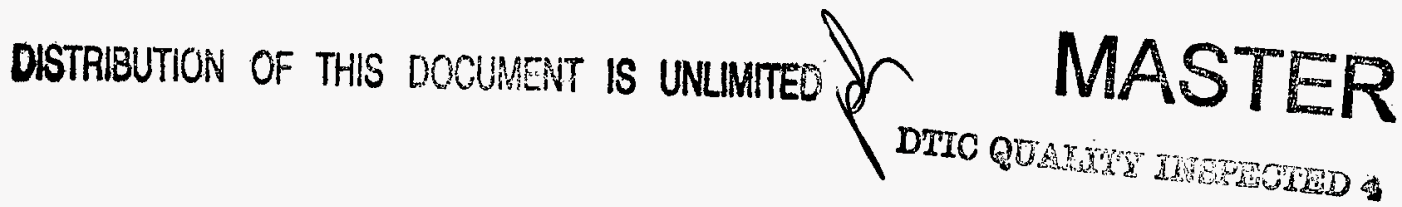




\section{DISCLAIMER}

This report was prepared as an account of work sponsored by an agency of the United States Government. Neither the United States Government nor any agency thereof, nor any of their employees, makes any warranty, express or implied, or assumes any legal liability or responsibility for the accuracy, completeness, or usefulness of any information, apparatus, product, or process disclosed, or represents that its use would not infringe privately owned rights. Reference herein to any specific commercial product, process, or service by trade name, trademark, manufacturer, or otherwise does not necessarily constitute or imply its endorsement, recommendation, or favoring by the United States Government or any agency thereof. The views and opinions of authors expressed herein do not necessarily state or reflect those of the United States Government or any agency thereof. 
2) Using this lattice constant, $\mathrm{MC}$ calculations at constant volume and hydrogen chemical potential are used to determine the hydrogen concentration. The hydrogen chemical potential is varied to obtain the desired hydrogen concentration (260 appm).

3) Using the same lattice constant and chemical potential for the cylindrical structure described above, $\mathrm{MC}$ calculations at constant volume and $\mathrm{T}=295 \mathrm{~K}$ are performed allowing hydrogen creation in a region near the dislocation core (see Figure 1).

A typical configuration in the $\mathrm{MC}$ calculation is then strained and relaxed (equivalent to $\mathrm{T}=0 \mathrm{~K})$ as in the case with no hydrogen.

\section{Results and Discussion}

The arrangement of the LCL-3 is shown in Figure 2. Here we see the two extended dislocations on intersecting slip planes move together to form the sessile stair rod dislocation $\delta \beta$ leaving two Shockley dislocations, $\mathrm{B} \delta$ and $\mathrm{D} \beta$. The configuration after relaxation with no applied stress is shown in Figure 3a. The situation is exactly as expected from dislocation theory, including the extent of the partial dislocation separation (7). Under application of an external hydrogen pressure of $190 \mathrm{~atm}$, which yields a far field hydrogen concentration of 260 appm at $295 \mathrm{~K}$, the MC calculation yields a typical configuration shown in Figure 3b. Here we see a very high hydrogen concentration in the regions near the partial dislocations. Due to the calculated high intrinsic stacking fault energy in the hydride, $400 \mathrm{~mJ} / \mathrm{m}^{2}$ vs. $100 \mathrm{~mJ} / \mathrm{m}^{2}$ in nickel, hydride does not form in the stacking fault regions between the partials. Closer examination of the high hydrogen concentration regions reveals that a stoichiometric hydride $(\mathrm{NiH})$ is formed.

Upon application of a uniaxial tensile stress (2.4\% strain) in the x-direction, (Figure 4a) in the absence of hydrogen, the right hand Shockley moves much closer to the stair rod dislocation and the left hand partial moves through the stair rod, leaving extrinsic stacking fault (7). In the presence of hydrogen the motion of the partial dislocations is severely limited at this stress level (Figure $4 \mathrm{~b}$ ). At a higher stress level (6.0\% strain), in the absence of hydrogen, the original right hand partial also moves through the stair rod (Figure 5a). Both left and right partials move a significant distance from the stair rod leaving a ribbon of both intrinsic and extrinsic stacking fault and the LCL has become inverted (7). The situation becomes more complicated (Figure 5b) in the presence of hydrogen: the partial originally on the left has moved through the stair rod similarly to the case of no hydrogen (Figure 5a), but the original right hand partial remains near its original position, similar to the situation shown in Figure 4a at a lower stress in the absence of hydrogen. In addition it appears that an additional partial dislocation has been emitted from the nickel/nickel hydride boundary. A likely reason that partials do not move away from the stair rod as easily in the hydrogen case is the large energy needed to form a stacking fault in the hydride. We summarize the transmission of partials through the stair rod in Table I. Clearly the presence of the hydrogen has significantly increased the uniaxial stress needed invert the stair rod.

Table I: Critical strain for absorption and emission of Shockley partial dislocation from stair rod.

\begin{tabular}{lll}
\hline & first partial $(\mathrm{B} \delta)$ & second partial $(\mathrm{D} \beta)$ \\
\hline no hydrogen & $2.3 \%$ & $4.8 \%$ \\
190 atm hydrogen & $4.7 \%$ & $>7 \%$ \\
\hline
\end{tabular}

In Figure 6 we examine the rearrangement of the hydrogen due to the applied uniaxial stress. It is clear that the motion of the partials has not significantly redistributed the 
hydrogen. Rearrangement of the hydrogen due to thermal motion was not considered here.

We have applied local strains up to $7 \%$ in the presence of hydrogen at which point the partial dislocation begins to strongly interact with the rigid boundary. No void nucleation or crack formation was observed in these atomistic models in contrast to the observations of void nucleation at slip band intersections in the Moody et al. (4) experiments. There is the possibility that void nucleation would occur at the lock in the presence of hydrogen if: 1) Thicker models were used. In order for a crack to nucleate in our models, it must do so through all of the thickness because of the periodic boundary conditions parallel to the LCL line. Using a thicker model the crack could perhaps nucleate in one small region and then spread along the axis.

2) The stress was applied at $\mathrm{T}>0$. It is possible crack nucleation is thermally activated.

3) The hydrogen was allowed to equilibrate in the stressed sample due to thermal motion. Hydrogen would move to the region of greatest tension and perhaps would enhance void nucleation.

4) Higher local strains were applied.

On the other hand, the fact that relaxation of the LCL is blocked by hydrogen, at least suggests that hydrogen causes the plastic relaxation of certain stress concentrations to become more difficult. Thus in the presence of hydrogen, plastic deformation is more difficult, leading to void formation as a mechanism to relax internal stress.

\section{Summary}

Calculations of the behavior of a LCL-3 under uniaxial tension were performed. Hydrogen was found to segregate strongly to the unstressed lock at room temperature and to severely limit the motion of the partial dislocations of the lock compared to the case with no hydrogen. The critical strains for lock rearrangement were increased by over a factor of two which suggests that hydrogen impedes plastic relaxation for this type of stress concentration. We did not, however, observe void formation or crack nucleation at the LCL.

\section{Acknowledgments}

This work was supported at Sandia National Laboratories by the Department of Energy under contract \#DE-AC04-94AL85000 and by the Office of Basic Energy Sciences, Division of Materials Research under Grant DE-FG06-87ER45287.

\section{References}

1. N. R. Moody, R. E. Stoltz \& M. W. Perra, Metal. Trans. A 18A, 1469-82 (1987).

2. N. R. Moody, S. L. Robinson \& J. W. M. Garrison, in Austenite superalloy selection and development for hydrogen service from a microstructural perspective, eds. Cialone, H. J., Johnson, G. W. E., Blum, M. E. \& Voort, G. F. V. (ASM, Metals Park, $\mathrm{OH})$, Vol. 16, pp. 17-92 (1988).

3. N. R. Moody, S. L. Robinson \& M. W. Perra, Eng, Fracture Mech. 39, 941-954 (1991).

4. N. R. Moody, M. I. Baskes, J. E. Angelo, P. V. Treeck \& R. M. Horn, to be submitted (1998).

5. A. H. Cottrell, AIME 212, 192 (1958).

6. J. P. Hirth, eds. Cahn, R. W. \& Haasen, P. (Elsevier, Amsterdam), pp. 1239 (1983). 
7. M. I. Baskes, R. G. Hoagland \& T. Tsuji, Modelling and Simul. in Mater. Sci. Eng. 6, 9-18 (1998).

8. J. E. Angelo, N. R. Moody \& M. I. Baskes, Modelling Simul. Mater. Sci. Eng. 3, 289-307 (1995).

9. M. I. Baskes, X. Sha, J. E. Angelo \& N. R. Moody, Modelling and Simul. in Mater. Sci. Eng. 5, 651-652 (1997).

10. M. S. Daw \& M. I. Baskes, Phys. Rev. Lett. 50, 1285-1288 (1983).

11. M. S. Daw \& M. I. Baskes, Phys. Rev. B 29, 6443 (1984).

12. M. S. Daw, S. M. Foiles \& M. I. Baskes, Mater. Sci. Rep. 9, 251-310 (1993).

13. M. I. Baskes, J. E. Angelo \& N. R. Moody, in Atomistic Calculations of Hydrogen Interactions with $\mathrm{Ni}_{3} \mathrm{Al}$ Grain Boundaries and $\mathrm{Ni} / \mathrm{Ni}_{3} \mathrm{Al}$ Interfaces, eds. Thompson, A. W. \& Moody, N. R. (The Minerals, Metals \& Materials Society, Warrendale, PA), pp. 77-90 (1996). 


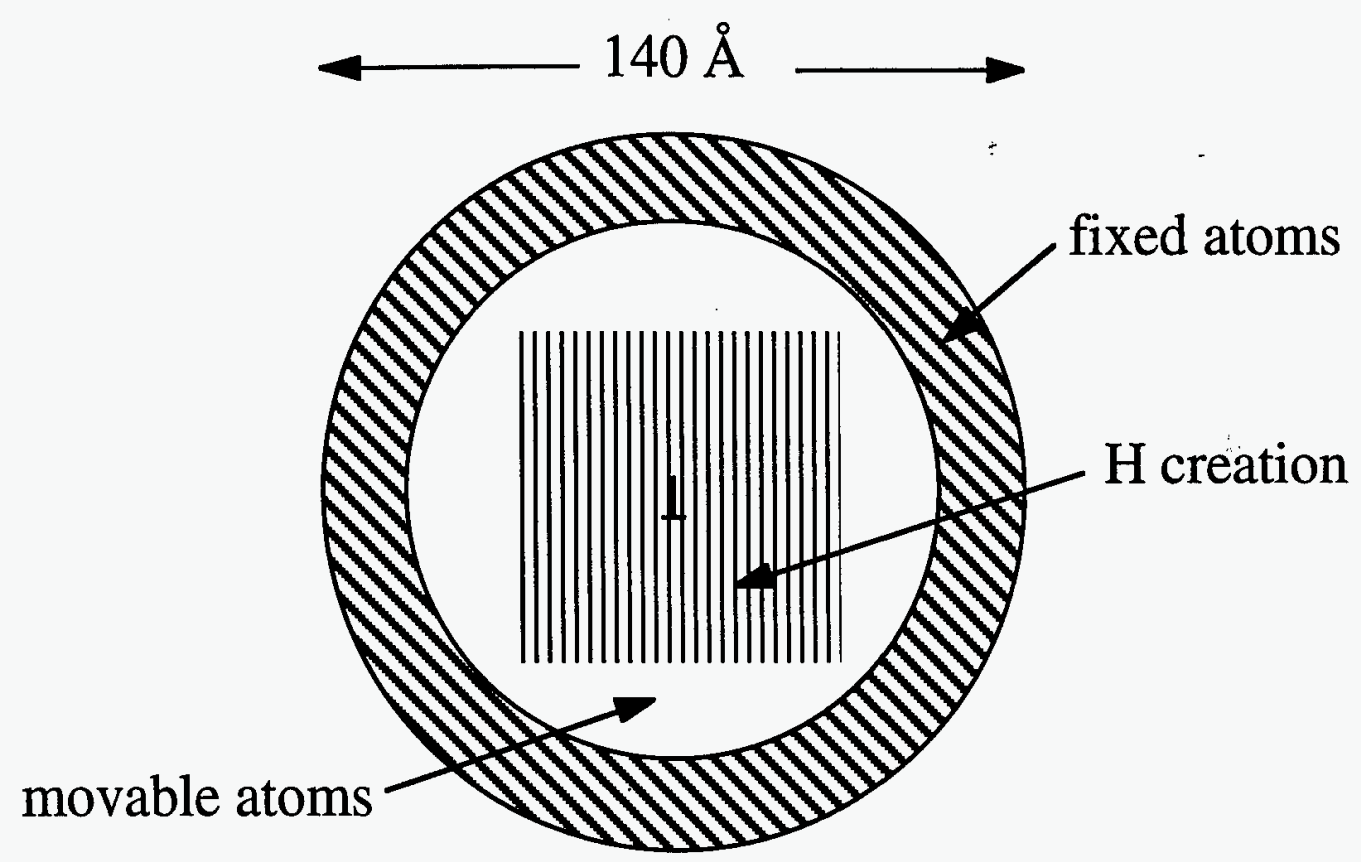

Figure 1: Geometry for atomistic calculations.

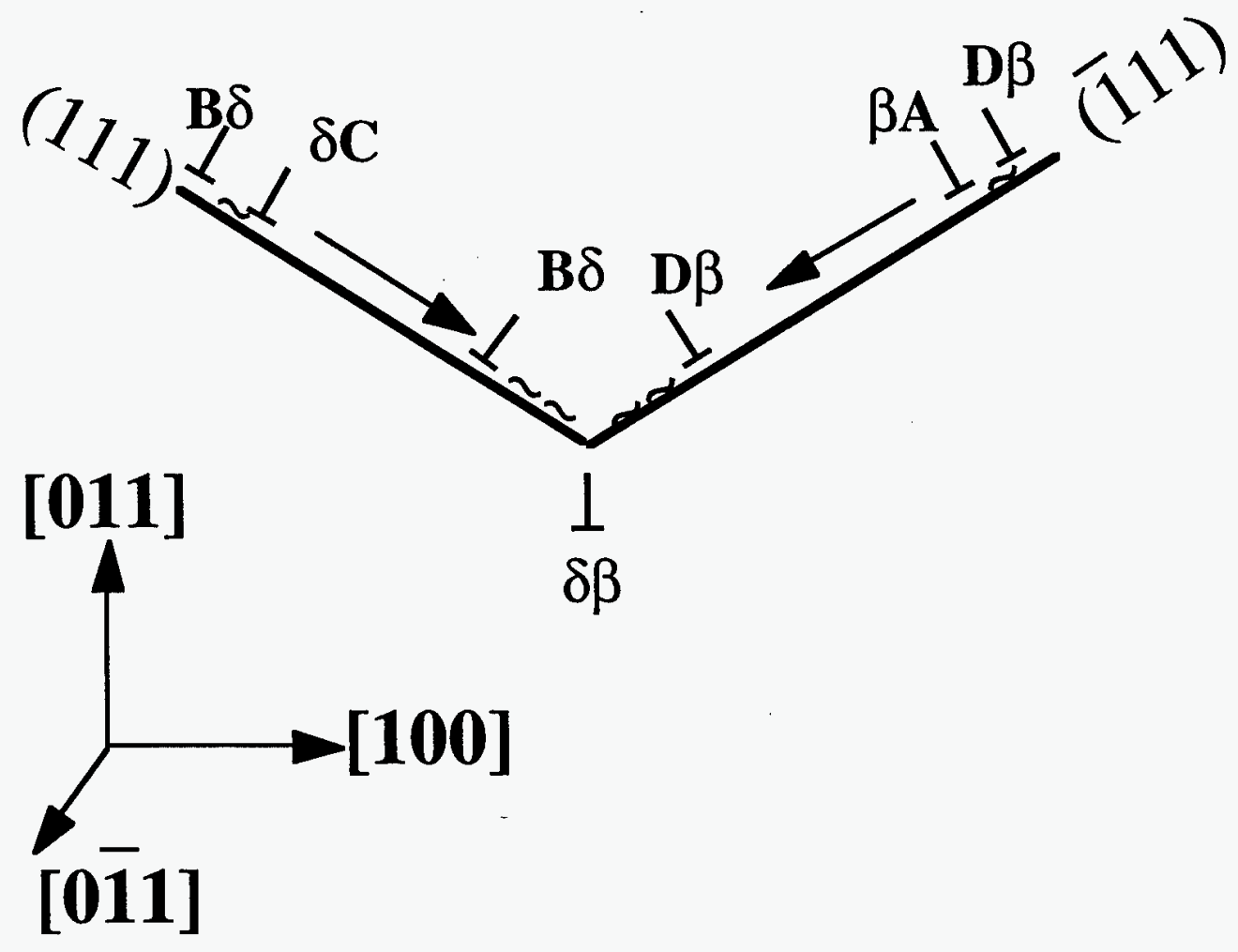

Figure 2: Formation of the LCL-3. Lattice dislocations on intersecting (111) planes dissociate into partial dislocations and then move together to form a stair rod partial dislocation, $\delta \beta$. 


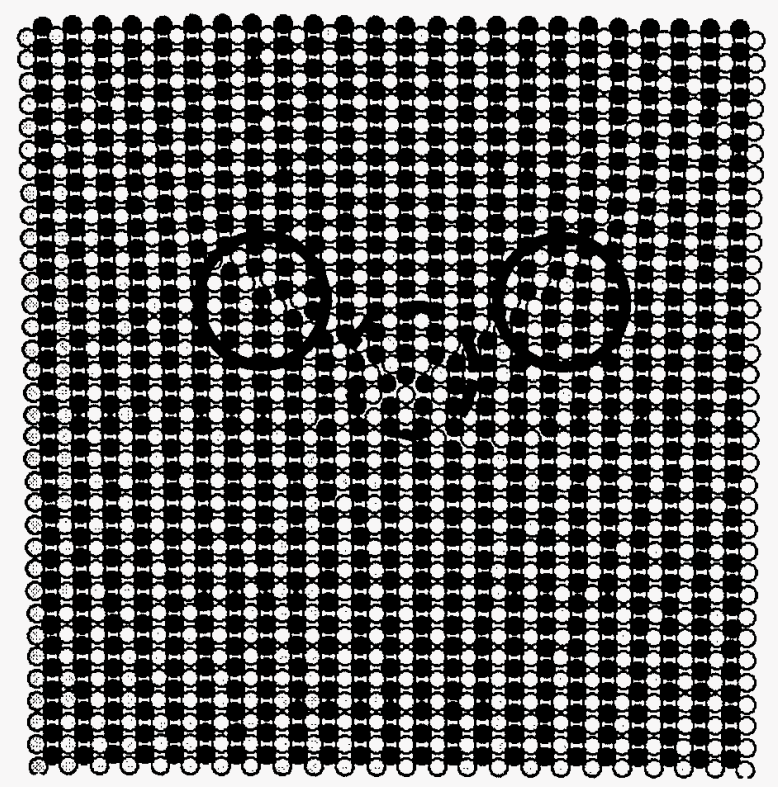

a)

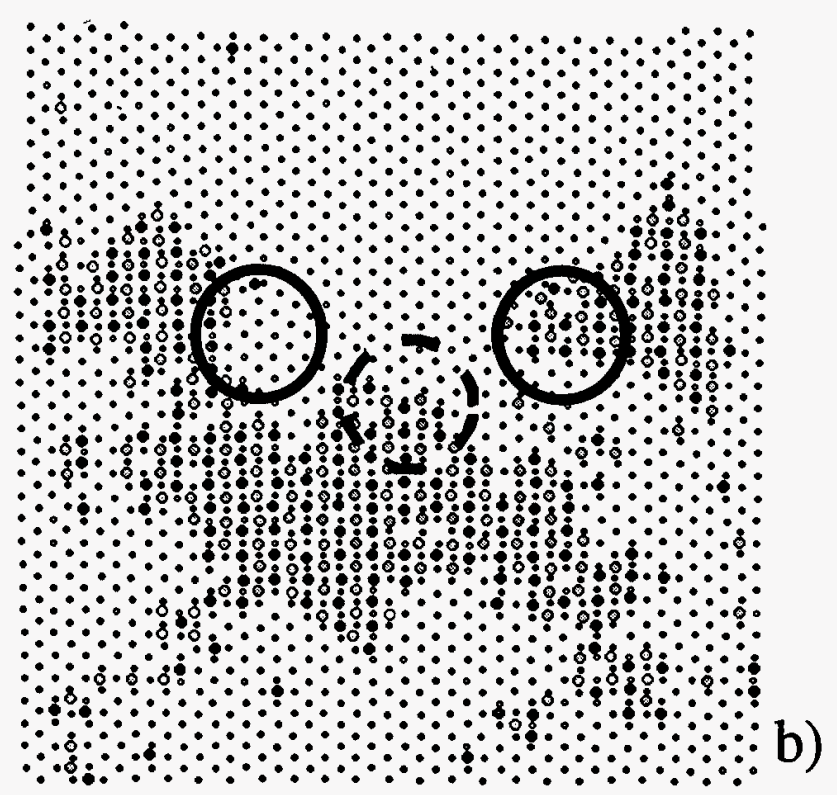

Figure 3: Central portion of the relaxed atomistic calculation with no applied stress for a) no hydrogen and b) hydrogen. The locations of the Shockley partial dislocations and stair rod dislocation are indicated by the large full and dashed circles respectively. In a) the nickel atoms are represented by black and white circles indicating different depths while in b) the hydrogen atoms are represented by the circles while the nickel atoms are represented by the small dots. Note that in the regions of very high hydrogen concentration the hydrogen lies in $\mathrm{O}_{\mathrm{h}}$ sites indicative of fully stoichiometric $\mathrm{NiH}$.
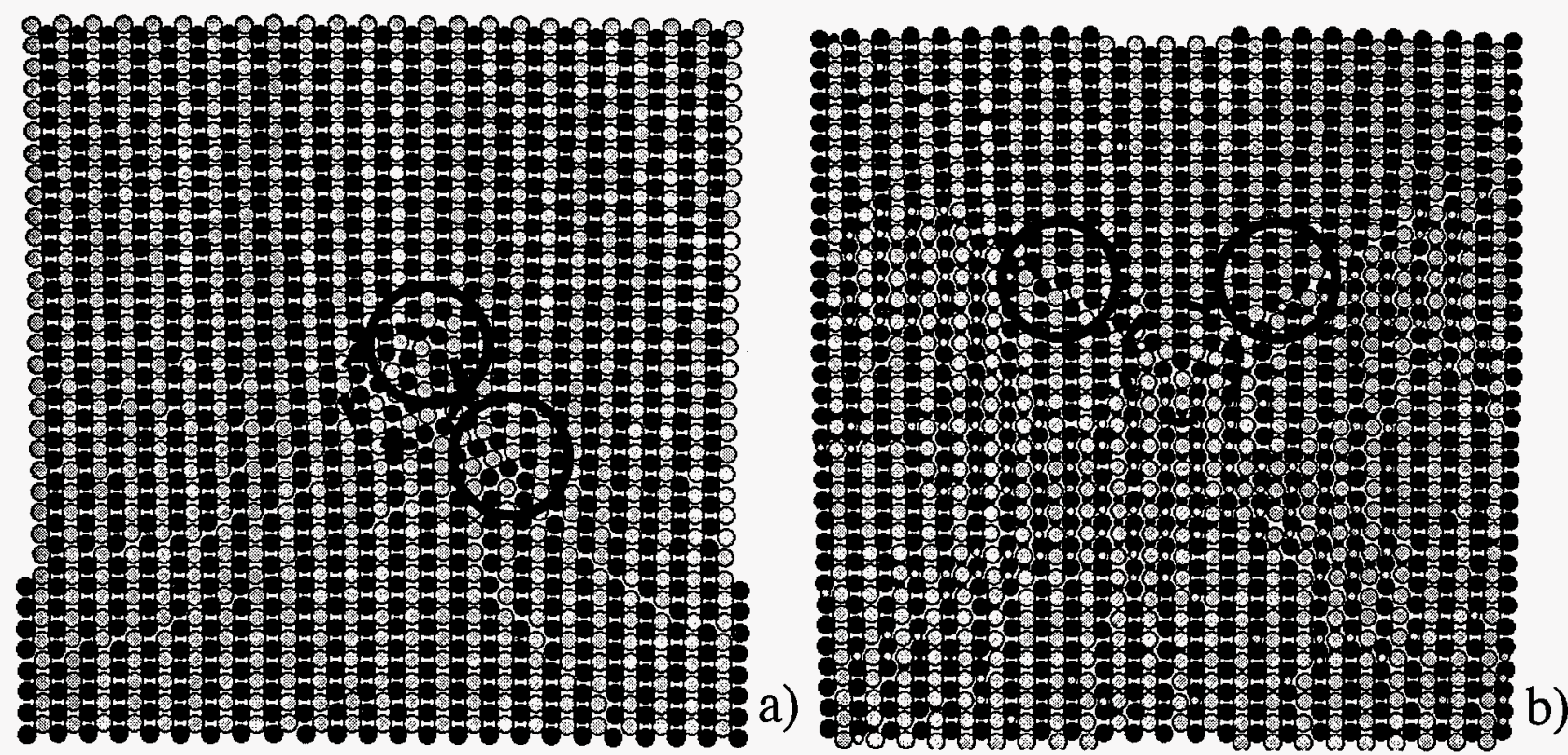

Figure 4: Central portion of the relaxed atomistic calculation with a uniaxial applied stress equivalent to a strain of $2.4 \%$ in the $x$-direction for a) no hydrogen and b) hydrogen. The locations of the Shockley partial dislocations and stair rod dislocation are indicated by the large full and dashed circles respectively. The nickel atoms are represented by black and white circles indicating different depths and in b) the hydrogen atoms are represented by the very small circles. In a) one of the Shockley partial dislocations has moved through the stair rod dislocation and the second Shockley dislocation has moved much closer to the stair rod dislocation. The presence of hydrogen inhibits this rearrangement of the partial dislocations. 

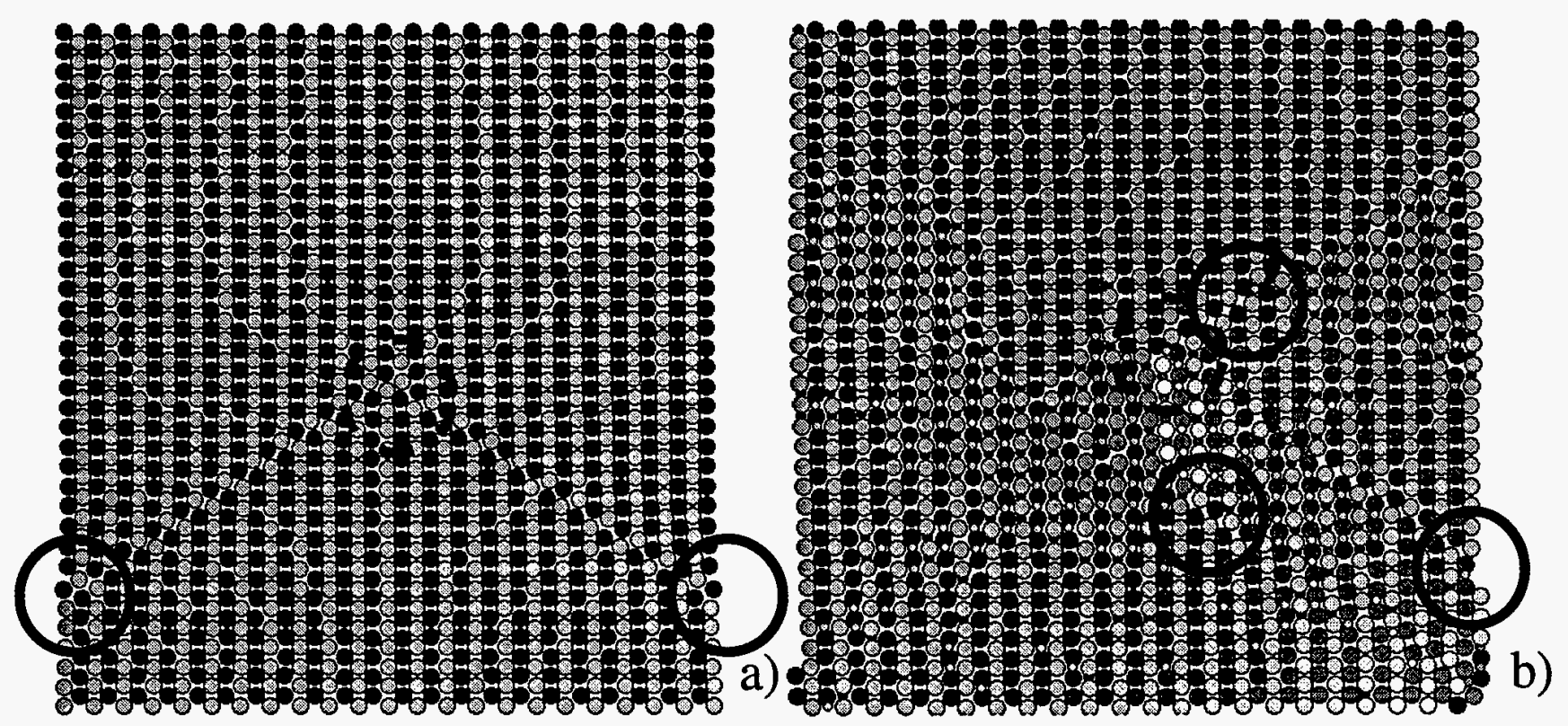

Figure 5: Central portion of the relaxed atomistic calculation with a uniaxial applied stress equivalent to a strain of $6.0 \%$ in the $x$-direction for a) no hydrogen and b) hydrogen. The locations of the Shockley partial dislocations and stair rod dislocation are indicated by the large full and dashed circles respectively. The nickel atoms are represented by black and white circles indicating different depths and in b) the hydrogen atoms are represented by the very small circles. In a) both of the Shockley partial dislocations have moved through the stair rod dislocation leaving a trail of intrinsic and extrinsic stacking fault. The presence of hydrogen has changed the rearrangement significantly with one partial moving much less and the stair rod emitting the dislocation just below the stair rod leaving a region with large shear.
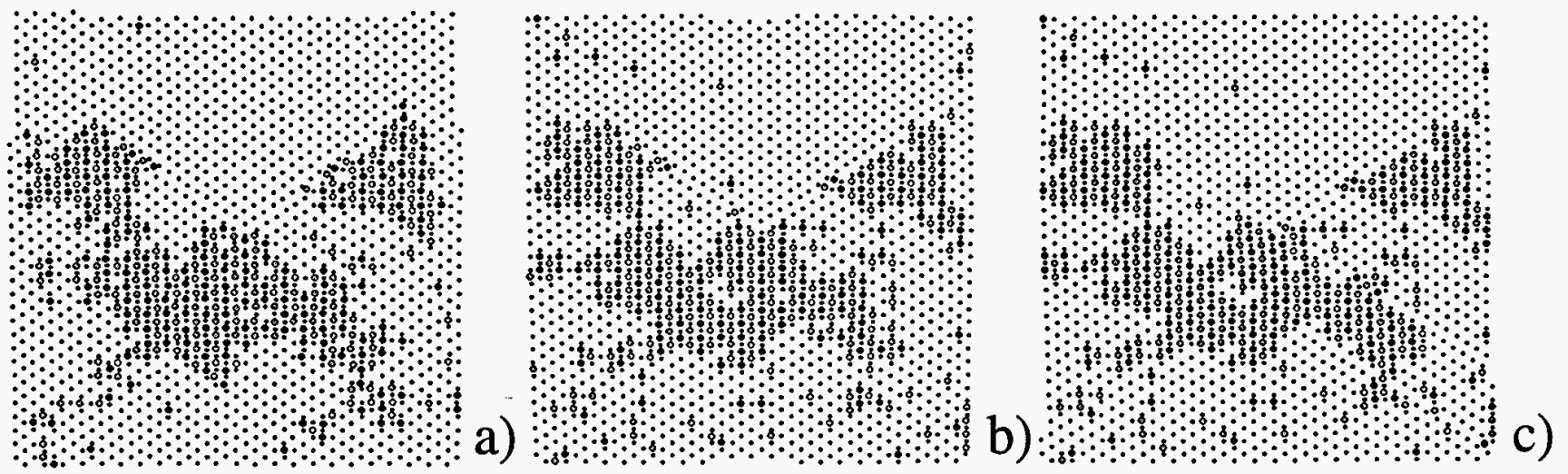

Figure 6: Central region of the simulation for a) $0 \%$, b) $2.4 \%$, and c) $6.0 \%$ strain in the $x$-direction. Small dots represent nickel atoms and larger dots represent hydrogen atoms. The distribution of hydrogen does not change significantly upon application of uniaxial tension. 


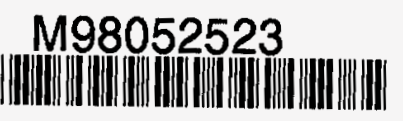

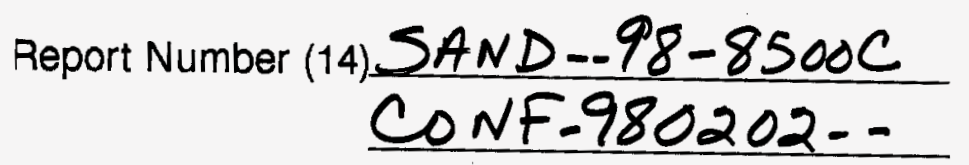

Jubl. Date (11) $\frac{199803 / 9}{D O E / E R, X F}$
sponsor Code (18)
JC Category (19) $U C-404$, DOE/ER 